\title{
用于可切换不对称催化的人工刺激响应催化体系
}

\author{
唐雨平 $a, b$ 何艳梅 $*, a$ 范青华 $*, a, b$ \\ ( ${ }^{a}$ 中国科学院化学研究所 中国科学院分子识别与功能重点实验室 北京分子科学国家研究中心 北京 100190) \\ $\left({ }^{b}\right.$ 中国科学院大学化学科学学院 北京 100049)
}

\begin{abstract}
摘要 受酶变构催化的启发, 人工刺激响应不对称催化体系的研究近年来愈来愈引起人们的兴趣. 通过将刺激响应性 官能团引入手性催化剂的设计, 利用光驱动、配位驱动、 $\mathrm{pH}$ 驱动以及氧化还原驱动等方式, 动态调控催化反应的活性 和对映选择性, 成功实现了多类可切换不对称催化反应. 主要介绍了近 20 年来人工刺激响应性不对称催化体系的发展 与研究现状. 通过选择代表性的研究成果, 从刺激响应模块与催化位点的有机结合和动态调控出发, 阐述其作为手性 开关催化剂或者立体发散性合成催化剂的设计原理、作用机制以及他们在不对称催化反应中的应用. 同时对这一新兴 领域的优势和局限进行总结, 并对其发展前景进行展望.
\end{abstract}

关键词＼cjkstart不对称催化; 刺激响应; 手性开关催化剂; 变构催化; 对映选择性发散催化

\section{Artificial Stimuli-Responsive Catalytic Systems for Switchable Asymmetric Catalysis}

\author{
Tang, Yuping ${ }^{a, b}$ He, Yanmei*,a $\quad$ Fan, Qinghua ${ }^{*, a, b}$ \\ ( ${ }^{a}$ Beijing National Laboratory for Molecular Sciences, CAS Key Laboratory of Molecular Recognition and Function, \\ Institute of Chemistry, Chinese Academy of Sciences (CAS), Beijing 100190) \\ ( ${ }^{b}$ School of Chemical Sciences, University of Chinese Academy of Sciences, Beijing 100049)
}

\begin{abstract}
Inspired by enzyme allosteric catalysis, the study on artificial stimuli-responsive asymmetric catalytic systems has attracted more and more attentions in recent years. In order to precisely control the catalytic activity and stereoselectivity, stimuli-responsive functionalities have been introduced into the catalyst design. A variety of asymmetric reactions featuring on/off-switchable catalysis and/or stereodivergent catalysis have been successfully achieved by using light-, coordination-, $\mathrm{pH}-$ and redox-driven chiral switchable catalysts. By selecting representative examples, the catalyst design principles, allosteric mechanism and their applications in switchable asymmetric reactions sre mainly introduced. At the same time, advantages and limitations of this emerging field are summarized, and perspectives for its future development are given.

Keywords asymmetric catalysis; stimuli-responsiveness; chiral switchable catalyst; allosteric catalysis; enantiodivergent catalysis
\end{abstract}

细胞内每时每刻都发生着不同的酶催化过程, 为了 保证每个生化反应之间互不干扰，生物体通常会利用反 馈机制和各种刺激响应机制来可逆调节酶的活性 ${ }^{[1]}$, 而 人工合成的催化剂通常只能在固定条件下催化反应 ${ }^{[2]}$. 近年来, 随着超分子化学的发展, 氢键、配位键和主-客 体相互作用等非共价键相互作用越来越多地被用来实 现催化反应中各组分间的预组装以及催化剂的设计合
成，促进了超分子催化 ${ }^{[3]}$ 这一前沿交叉学科的蓬勃发展. 至今，化学家们已报道了大量超分子催化新体系 ${ }^{[4]}$, 其 中许多催化模式与酶催化具有很大的相似性. 因此, 利 用超分子手段模拟酶催化反应的变构调控机制，将刺激 响应模块引入催化剂的设计, 发展刺激响应性智能催化 体系就成为科学家不断追求的目标.

近年来, 已报道了许多刺激响应性智能催化体系 ${ }^{[5]}$.

\footnotetext{
* Corresponding authors. E-mail: heym@iccas.ac.cn; fanqh@iccas.ac.cn

Received June 30, 2020; revised August 3, 2020; published online August 11, 2020.

Dedicated to the 40th anniversary of Chinese Journal of Organic Chemistry.

Project supported by the National Natural Science Foundation of China (Nos. 21772204, 21521002), and the Key Research Program of Frontier Sciences, Chinese Academy of Sciences (No. QYZDJ-SSW-SLH023).

国家自然科学基金(Nos. 21772204, 21521002)和中国科学院前沿科学重点研究(No. QYZDJ-SSW-SLH023)资助项目.
} 
通过从外部输入物理(光/热、机械力)和/或化学刺激信号 (酸碱、离子和中性分子), 实现了多种刺激响应性的可 切换催化反应. 这些智能催化剂都具有如下的特点: 外 界刺激信号的输入能够(可逆)改变催化剂的组成和/或 结构, 从而在催化反应中表现出 “开关” 的性质, 即(可 逆)调控催化反应活性和选择性, 使催化反应活性在高 与低之间切换, 或给出立体选择性不同或完全相反的产 物.

不对称催化是制备各种手性化合物的最为高效绿 色的途径, 一般来说, 制备一对对映异构体需要使用绝 对构型相反的两种催化剂, 增加了合成上的繁琐和困 难. 发展简单高效的催化体系 ${ }^{[6]}$ 以制备一对对映异构体 对于手性药物研究至关重要 ${ }^{[7]}$. 将刺激响应性官能团引 入手性催化剂的设计, 为通过外部刺激实现手性催化剂 对映选择性的改变提供了全新的思路. 近年来, 人工刺 激响应手性催化体系已成为不对称超分子催化研究的 新增长点 ${ }^{[8]}$, 愈来愈引起人们的兴趣. 本综述主要介绍 近 20 年来人工刺激响应性不对称催化体系的发展与研 究现状. 通过选择代表性的研究成果, 从刺激响应模块 与催化位点的有机结合和动态调控出发, 阐述其作为手 性开关催化剂或者立体发散性合成催化剂的基本策略 以及他们在不对称催化反应中的应用. 本文将以驱动方 式作为主线, 从光/热驱动、配位驱动、 $\mathrm{pH}$ 驱动和氧化 还原驱动四个方面进行叙述, 但不包括溶剂对催化剂 结构的影响以及聚合物型刺激响应手性开关催化剂的 内容 ${ }^{[9]}$.

\section{1 光驱动的手性开关催化体系}

光作为一种非入侵式的刺激信号, 具有在时间和空 间上精准可控的优点, 不会在催化系统中累积, 同时可 以通过选定特定的波长和发色团与催化活性位点正交. 近年来, 通过在催化剂结构中引入光刺激响应基团(如 偶氮苯等), 化学家们已发展了多种光驱动刺激响应催 化剂体系 ${ }^{[10]}$.

1,2-二噻吩基乙烯是一类典型的光致异构基团 ${ }^{[11]}$, 在不同波长光的照射下可以进行可逆的关环和开环反 应. 不同于偶氮苯官能团, 这些异构体的热稳定性良好, 加热并不会对这一可逆的结构变化产生影响. 2005 年, Branda 小组 ${ }^{[12]}$ 报道了首例基于 1,2 -二噻吩基六氟环戊烯 骨架的光驱动手性金属催化剂(Scheme 1). 通过在两个 噻吩基的邻位引入手性噁唑啉基团, 他们合成了双噁唑 啉手性配体, 通过光照能够在 State I 和 State II 之间进行 可逆切换. 作者选用苯乙烯的环丙烷化作为模板反应, 当处于 State I 时, 形成分子内双齿配位金属催化剂, 能 够得到中等的对映选择性, 而光致关环催化物种(State
II)相当于单齿配位，得到几乎消旋的产物.

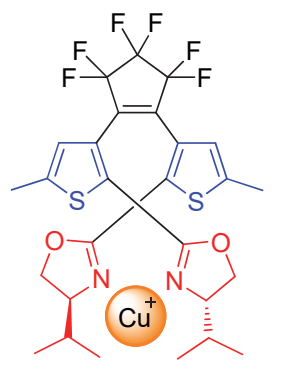

$1 \mathbf{a}$

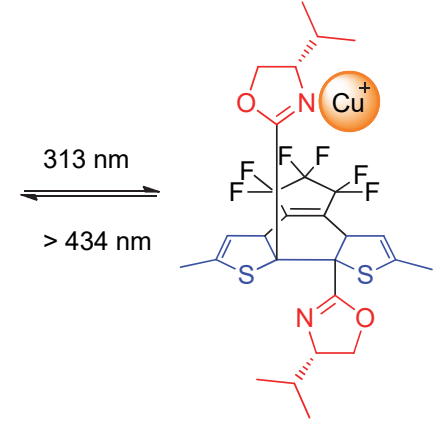

$1 \mathrm{~b}$
State I (high selectivity)

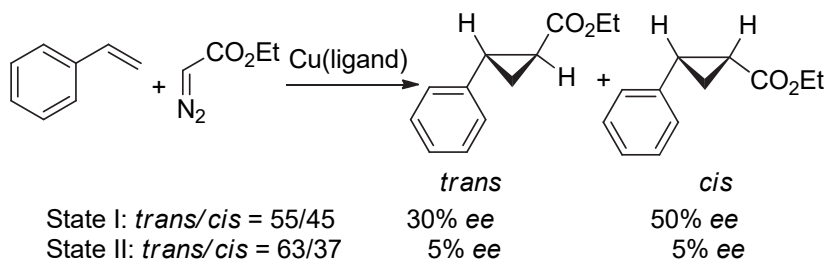

图式 1 Branda 小组发展的基于 1,2 -二噻吩基乙烯的光驱动催 化剂及其催化的可切换不对称环丙烷化反应

Scheme 1 A photo-driven catalyst based on 1,2-dithienylethylene and its application in switchable asymmetric cyclopropanation reactions developed by Branda group

具有刚性二苯乙烯骨架的四取代烯烃光致异构化 合物, 因其异构体几何构型差异大, 且具有良好的量子 效率和热稳定性, 已被广泛应用于光驱动分子机器和分 子力探针的研究. 1999 年 Feringa 等 ${ }^{[13]}$ 基于刚性四取代 烯烃骨架, 创制了第一例光/热驱动单方向 $360^{\circ}$ 旋转分 子马达. 随后, Feringa 小组 ${ }^{[14]}$ 将这一分子马达的设计理 念应用于构建光刺激响应的智能手性催化剂, 取得了系 列引人注目的研究成果.

2011 年, 通过在第一代手性联二狮满分子马达的 转子和定子上分别引入对二甲基氨基吡啶和硫脲有机 小分子协同催化位点，他们 ${ }^{[15]}$ 合成了光/热驱动的 $360^{\circ}$ 单方向旋转的手性分子马达双功能催化剂(Scheme 2), 通过精准控制马达的旋转, 可以得到四种不同的催化剂 状态(State I、State II、State III 和 State IV), 并将其应用 于邻甲氧基硫酚对环已烯酮的不对称 Michael 加成反 应. 由于这四种立体异构体的顺反结构或/和螺旋手性 存在不同，造成其催化中心手性环境的不同，且两个协 同催化中心的相对位置也存在远近的差异, 从而在不对 称催化中表现出活性和对映选择性的显著差别. 其中, State I 催化剂得到消旋产物, 反应活性也很低, $15 \mathrm{~h}$ 收 率仅为 7\%, 这是因为催化剂为反式构型, 两个催化位 点相距较远，不利于分子内的协同催化及手性控制; State II 催化剂得到 $S$ 构型产物 $(50 \% e e)$, 收率为 $50 \%$; State III 催化剂得到 $R$ 构型产物( $54 \% e e)$, 收率为 $83 \%$. 
<smiles>Cc1cc(-c2ccc(NC(=S)Nc3cc(C(F)(F)F)cc(C(F)(F)F)c3)cc2)c(C)c2c1C[C@H](C)C2=C1c2c(C)c(-c3ccc(Nc4cc(N(C)C)ccn4)cc3)cc(C)c2C[C@H]1C</smiles>

$(R, R)-(P, P)-$ trans-2 State I (OFF) $-10^{\circ} \mathrm{C}$ step 4<smiles>Cc1cc(-c2ccc(Nc3cc(N(C)C)ccn3)cc2)cc(C)c1/C(=C1/c2c(C)cc(-c3ccc(NC(=S)Nc4cc(C(F)(F)F)cc(C(F)(F)F)c4)cc3)c(C)c2C[C@H]1C)C(C)C</smiles>

$(R, R)-(M, M)-$ trans-2 State IV (unstable)<smiles>O=C1C=CCCC1</smiles><smiles>COc1ccccc1S</smiles>

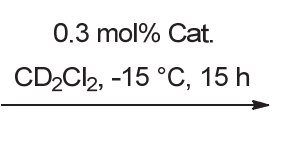

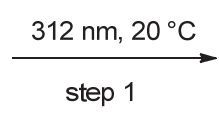

$\frac{312 \mathrm{~nm},-60^{\circ} \mathrm{C}}{\text { step } 3}$<smiles>COc1ccccc1SC1CCCC(=O)C1</smiles>

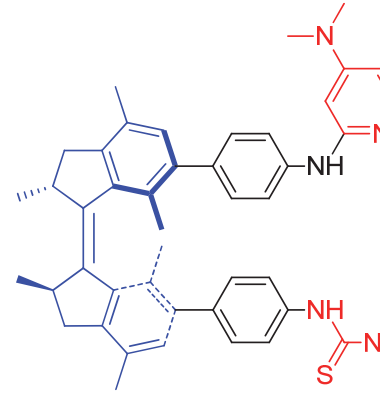

$(R, R)-(M, M)-c i s-2$ State II (ON and S-selectivity)<smiles>O=[N+]=C1C=C(C(F)(F)F)C=C(C(F)(F)F)C1</smiles>

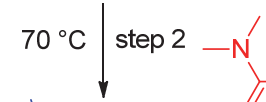<smiles>CNC(=S)Nc1ccc(-c2cc(C)c3c4c2Cc2c(-c5ccc(Nc6ccccn6)cc5)cc(C)c5c2C(=C3[C@@H](C)C5)C4C)cc1</smiles>

$(R, R)-(P, P)-c i s-2$ State III (ON and $R$-selectivity)<smiles>COc1cc(NC(C)=S)cc(C(F)(F)F)c1</smiles>

State I: OFF State, racemic, $7 \%$ yield

State II: S-form, $50 \%$ ee, $50 \%$ yield State III: $R$-form, $54 \%$ ee, $83 \%$ yield

图式 2 Feringa 小组发展的基于第一代分子马达的双功能有机小分子催化剂及其催化的可切换不对称 Michael 加成反应 Scheme 2 A bifunctional organic catalyst based on the first generation molecular motor and its application in switchable asymmetric Michael addition reaction developed by Feringa group

而 State IV 由于不稳定，所以没有进行催化反应的尝试. 上述工作实现了由单一对映异构体手性催化剂制备绝 对构型相反的手性产物. 有意思的是，作者发现分子马 达的旋转方向和螺旋手性是由转子和定子上的碳中心 手性决定的.

随后，他们希望探索这一光驱动手性分子马达催化 剂的普适性，将其应用拓展到其他的不对称催化反应. 但是，在初步尝试 2,2,2-三氟苯乙酮与硝基甲烷的不对 称 Henry 反应时, 催化剂 $(R, R)-(M, M)$-cis-2 只能给出消 旋产物, 且产率小于 $10 \%$. 于是, 他们着手设计合成新 型手性分子马达催化剂(Scheme 3$)^{[16]}$. 去除催化中心与 分子马达转子和定子间的苯基连接臂, 使得两个协同催 化中心进一步靠近, 可以提升其协同催化效率, 同时有 利于实现产物的手性诱导. 以此新型手性分子马达催化 剂再次尝试不对称 Henry 反应时, 催化剂处于 State II 时, 能以 $93 \%$ 的收率得到 $e e$ 值为 $72 \%$ 的 $R$ 构型产物; 处 于 State III 时, 可以 $92 \%$ 的收率得到 $S$ 构型产物( $42 \%$ $e e)$; 而催化剂处于 State I 时仅给出少量的消旋产物. 这 一手性分子马达催化剂在其他 $\alpha, \alpha, \alpha$-三氟甲基芳基酮和 $\alpha, \alpha, \alpha$-三氟甲基烷基酮与硝基甲烷的不对称 Henry 反应 中也表现出较高的催化活性和一定的对映选择性.
具有 $C_{2}$-对称轴的手性双齿膦配体是一类优秀手性 配体，被广泛应用于金属催化的不对称反应研究. 基于 分子马达在有机小分子不对称催化方面的成功应用, Feringa 小组进一步将其拓展至金属催化领域. 他们 ${ }^{[17]}$ 参考 Trost-配体的分子设计, 选用酰胺基团作为连接臂, 将三苯基膦引入手性分子马达的定子和转子上, 分别合 成了具有相反酰胺键连接方式的手性分子马达双膦配 体 4a 和 4b (Scheme 4) ${ }^{[18]}$, 在后续的光/热驱动分子马达 配体单方向旋转的测试中, 发现只有 $\mathbf{4 b}$ 可以成功实现 前述分子马达的 “四态” 旋转功能. 随后，他们选择钯 催化内消旋-2-环戊烯-1,4-二醇酯的去对称化为模板反 应, 详细研究了三种催化剂异构体的催化效果. 与预期 的结果一致，当配体处于 State II 状态时，以 $90 \%$ 的收率 得到 $86 \%$ ee 的 $(3 R, 4 S)$-手性噁唑烷酮产物; 处于 State III 状态时则以 $85 \%$ 的收率给出 $(3 S, 4 R)$-手性产物 $(88 \% e e)$; 而配体处于 State I 状态时，仅以 $65 \%$ 的收率得到消旋产 物. 与分子马达有机小分子催化剂不同, 由于钯/分子马 达双膦配体配合物的稳定性较高, 光/热原位驱动其异 构化的结果比较复杂，使得反应的选择性显著下降。

三氮唑基团是一类可以替代酰胺基团的阴离子受 体，其 5-位碳原子上电正性的氢原子可与多种阴离子形 


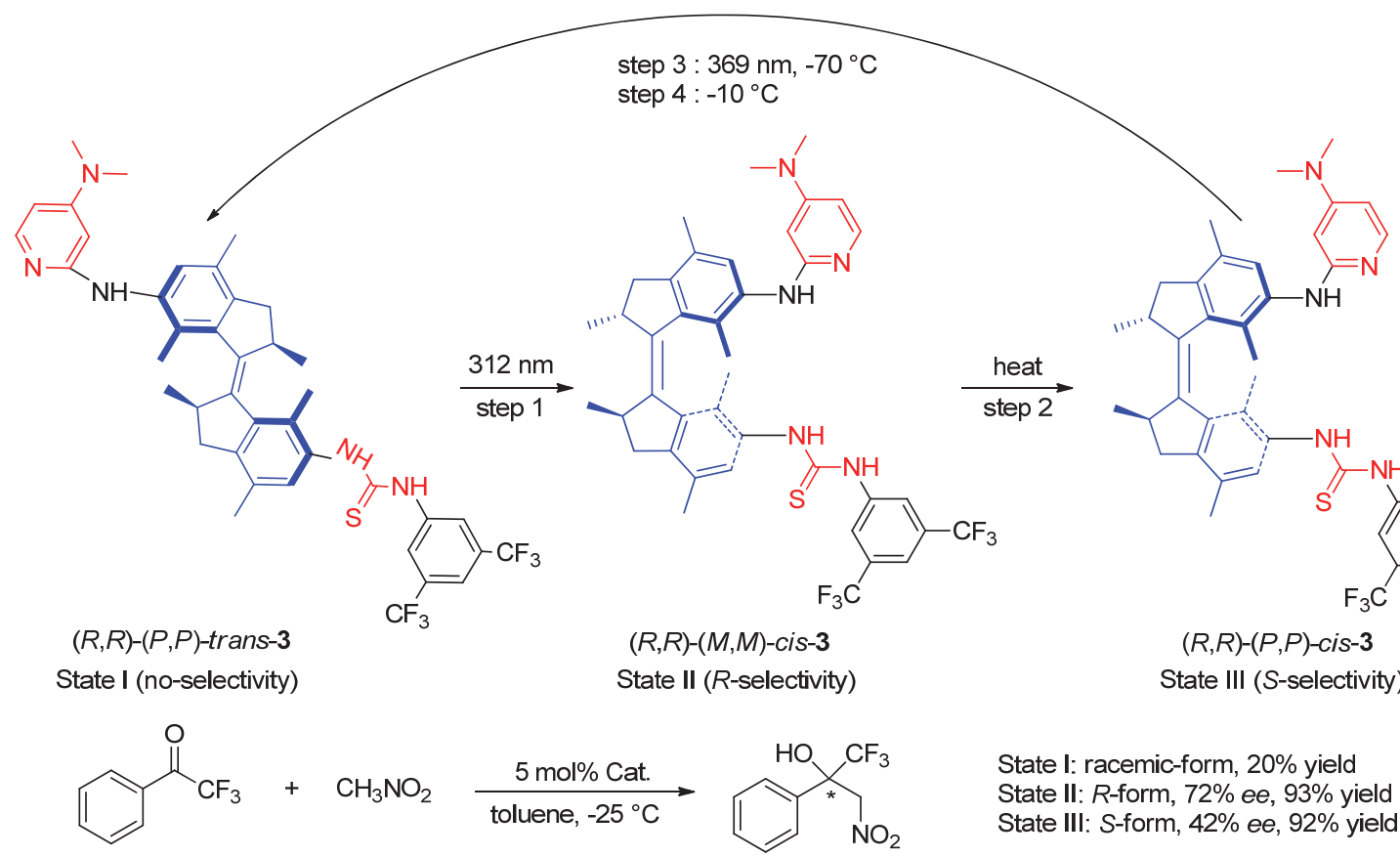

图式 3 Feringa 小组发展的基于第一代分子马达的高活性双功能有机小分子催化剂及其催化的可切换不对称 Hennry 反应 Scheme 3 Highly active bifunctional organic catalyst based on the first generation molecular motor and its application in switchable asymmetric Henry reactions developed by Feringa group

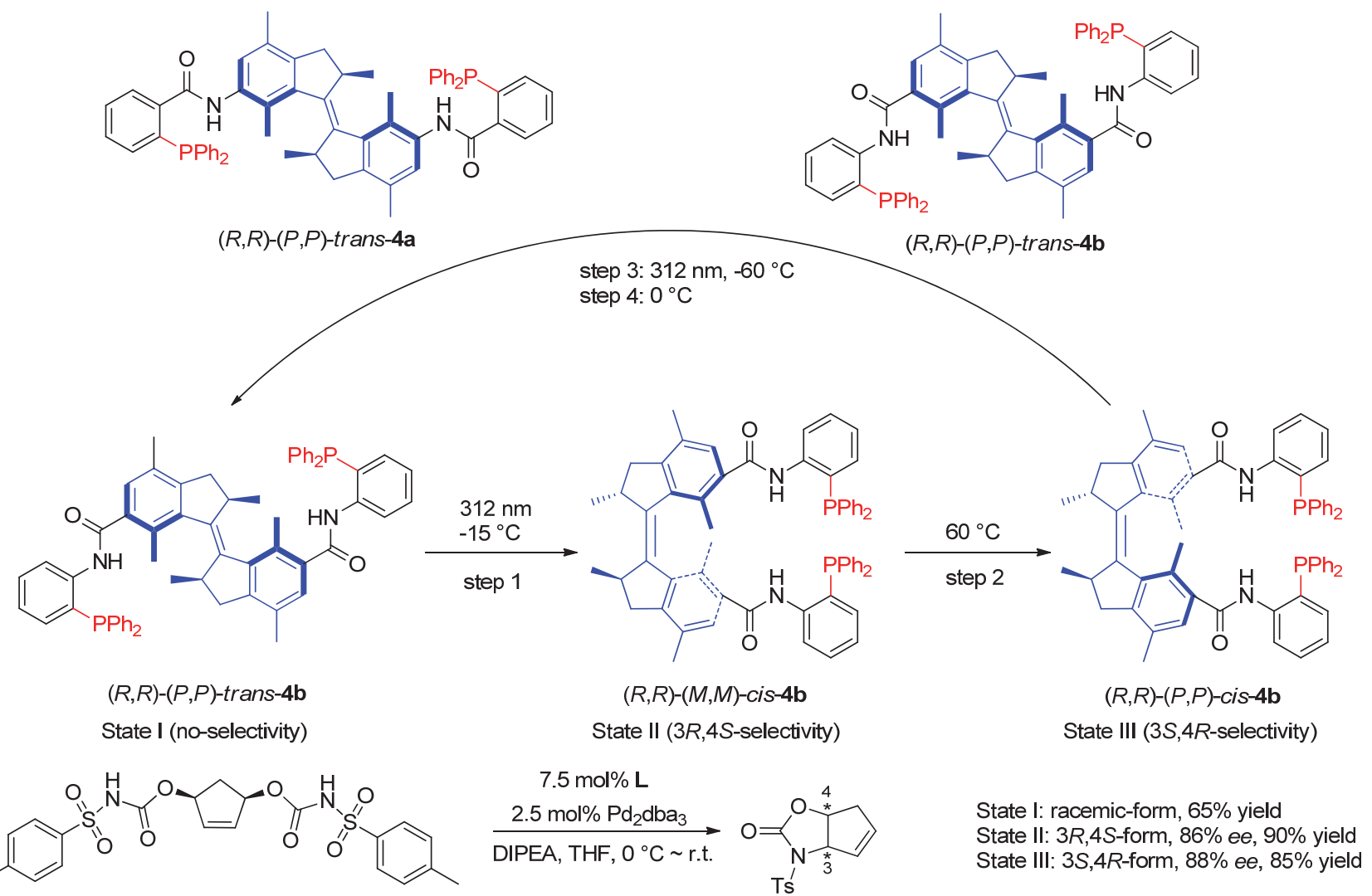

图式 4 Feringa 小组发展的基于第一代分子马达的钯/双膦金属催化剂及其催化的可切换不对称催化反应

Scheme 4 A Pd/bisphosphine catalyst based on the first generation molecular motor and its application in switchable asymmetric reactions developed by Feringa group

成氢键; 更为重要的是, 芳基三氮唑低聚物可与 $\mathrm{Cl}^{-}$形 成螺旋超分子组装体 ${ }^{[19]} .2020$ 年, Feringa 小组 ${ }^{[20]}$ 将芳基 
三氮唑基团引入到第一代光/热驱动手性分子马达的定 子和转子上, 发展了可调控的不对称阴离子结合催化剂 (asymmetric anion-binding catalyst)(Scheme 5). 通过光/ 热驱动可以精准控制马达的不同状态(State I, State II 和 State III), 研究发现 State II 和 State III 均可与 $\mathrm{Cl}^{-}$形成 1：1 的超分子手性螺旋组装体, 但螺旋手性构型相反. 在硅基烯酮缩醛对 1-氯-异色满衍生物的亲核加成反应 中, 螺旋手性相反的 cis-分子马达催化剂给出绝对构型 相反的手性产物, 两种手性产物间最大对映体过量差值 $(\Delta e e)$ 达到 $142 \%$. 值得注意的是, 由于溶解度差, 相同 条件下 State II 状态分子只能催化 1-氯-异色满衍生物的 部分转化, 而 State I 状态分子只能得到消旋的产物.

最近, Feringa 小组 ${ }^{[21]}$ 将他们发展的第二代分子马达 用来构筑新型的人工刺激响应手性催化体系. 与第一代
分子马达不同，第二代分子马达旋转过程中存在亚稳态 异构体，为此他们发展出光驱动的 “两态” 手性分子开 关催化剂 ${ }^{[22]}$. 他们将联苯二酚单元引入到分子开关的 骨架中，发现碳手性中心能够诱导分子开关螺旋手性的 生成，并进一步诱导产生联苯二酚的单一轴手性. 同时, 在不同波长光的照射下，可以使稳定的 State I 与其半稳 态 State II 之间发生可逆转化(Scheme 6) ${ }^{[23]}$. 以此手性分 子开关为配体，在二乙基锌对苯甲醛衍生物的不对称加 成反应中，两种状态配体(State I 和 State II)分别得到绝 对构型相反的手性醇产物, 且其 $e e$ 值的差异最大可达 $113 \%$.

随后，他们 ${ }^{[24]}$ 将含有联苯二酚的手性分子开关进 一步衍生化，合成了系列单齿亚磷酰胺配体(Scheme 7), 发现氮原子上取代基的不同会直接影响亚磷酰胺配体

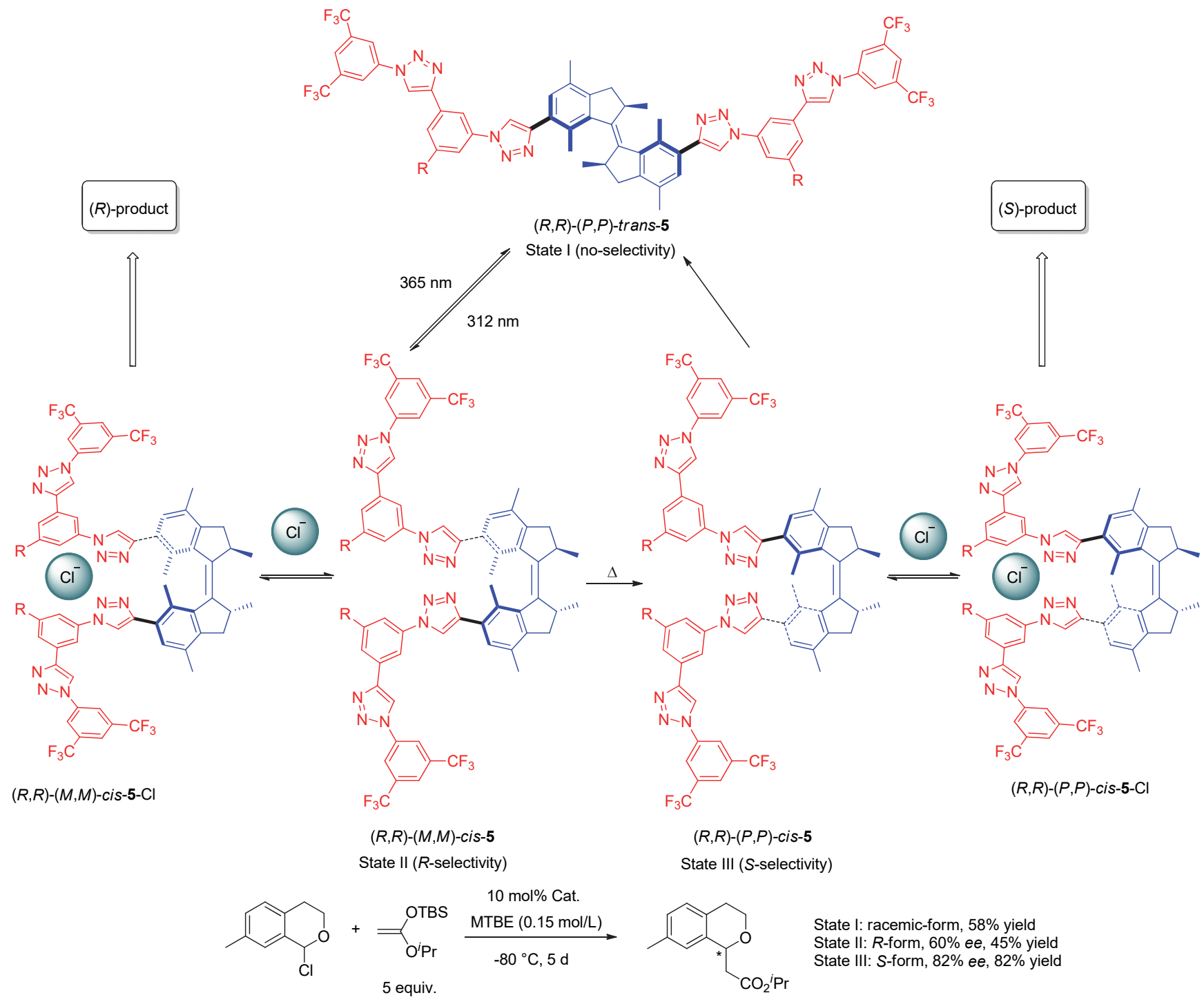

图式 5 Feringa 小组发展的基于第一代分子马达的阴离子结合催化剂及其催化的可切换不对称催化反应

Scheme 5 An anion-binding catalyst based on the first generation molecular motor and its application in switchable asymmetric reactions developed by Feringa group 


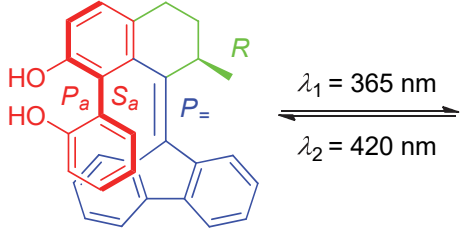

$\left(R, P_{=}, P_{a}, S_{a}\right)-6$

State I (R-selectivity)
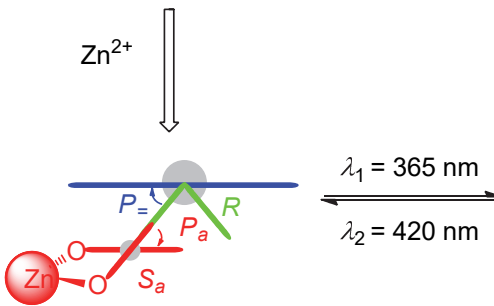

$\mathrm{Zn}-\left(R, P_{=}, P_{a}, S_{a}\right)-6$

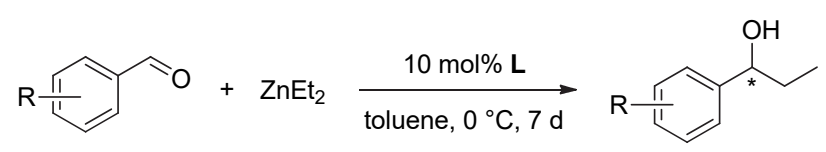

State I: $R$-form, up to $68 \%$ ee, up to $87 \%$ yield State II: $S$-form, up to $55 \%$ ee, up to $87 \%$ yield

图式 6 Feringa 小组发展的基于第二代分子马达的手性联苯 二酚配体及其催化的可切换不对称催化反应

Scheme 6 Chiral diphenol ligand based on the second generation molecular motor and its applicaton in switchable asymmetric reactions developed by Feringa group

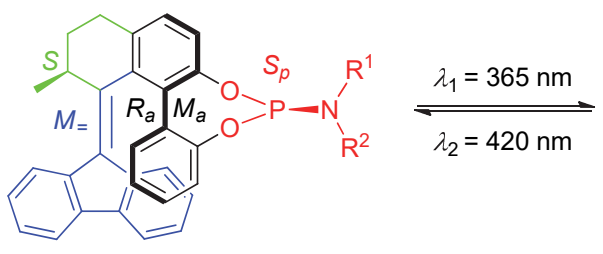

$\left(S, S_{p}, M_{=}, M_{a}, R_{a}\right)-7$

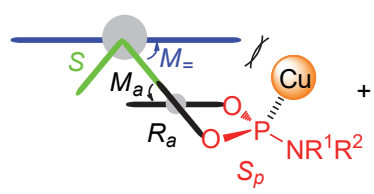

$\mathrm{Cu}-\left(S, S_{p}, M\right)-7$

Low activity, low selectivity for (S)-product

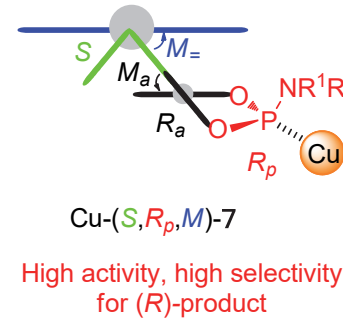

State I ( $R$-selectivity)<smiles>CCOC(C)=[C+]1C=CCCC1</smiles>

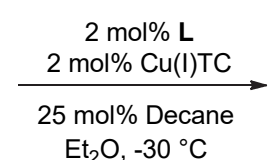<smiles>CCC1CCCC(=O)C1</smiles>

$N R^{1} R^{2}=$
中所含一对非对映异构体(磷手性区别)的物质的量比 值，并证实了其可逆光异构化过程的存在(光异构化仅 影响分子的螺旋手性), 即从 State I 可逆切换到 State II, 且铜盐的存在并不能对这一过程产生影响. 在铜催化二 乙基锌对环已烯酮的不对称共轭加成反应中，所有配体 均显示了较高的化学选择性, 以良好的收率得到 1,4-加 成的手性酮产物. 利用这样一对非对映异构体催化剂在 光照条件下的可逆转化, 能够实现加成产物的手性翻 转, $\Delta e e$ 高达 $106 \%$. 而动力学实验结果表明, 产物对映 选择性的高低是由光控分子开关的动态螺旋手性与磷 中心手性之间匹配度的高低决定的.

采用与 Feringa 小组相似的策略, 基于自己发展的 $C_{2}$-对称的手性二苯并环庚烷结构的光响应分子开关 ${ }^{[25]}$, 2017 年 $\mathrm{Chen}$ 和 $\mathrm{Yu}$ 等 ${ }^{[26]}$ 报道了一例含有 4-氨基吡啶催 化中心的新型螺旋手性开关催化剂(Scheme 8). 通过光/ 热刺激可以获得一对螺旋手性不同的非对映异构体 (State I 和 State II), 他们在催化 $O$-酰基唤酯的不对称 Steglich 重排反应中, 可以高产率、高对映选择性地分别 得到绝对构型相反的手性季碳产物 [最高为 $91 \%$ ee 的 $(R)$ 构型产物和 $94 \% e e$ 的 $(S)$ 构型产物], 这是目前光响应 手性催化剂中效果最好的. 作者随后通过对 State I 催化 剂参与的离子对过渡态的密度泛函理论(DFT)计算, 证 实较大的 HOMO-LUMO 相互作用和极小的位阻有利于 发生顺式的 $R e$-面进攻，进而生成 $(R)$-手性产物.

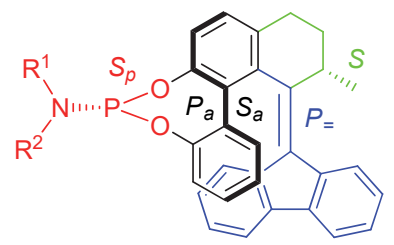

$\left(S, S_{p}, P_{=}, P_{a}, S_{a}\right)-7$

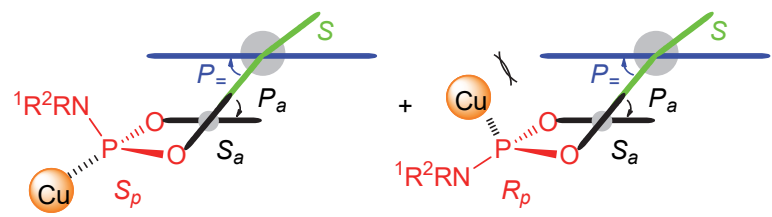

$\mathrm{Cu}-\left(S, S_{p}, P\right)-7$

High activity, high selectivity for (S)-product

$\mathrm{Cu}-\left(S, R_{p}, P\right)-7$

Low activity, low selectivity for $(R)$-product

State II (S-selectivity)

图式 7 Feringa 小组发展的基于第二代分子马达的手性亚磷酰胺配体及其在可切换不对称催化反应中的应用 Scheme 7 Chiral phosphoramidite ligands based on the second generation molecular motor and their application in switchable asymmetric reactions developed by Feringa group 


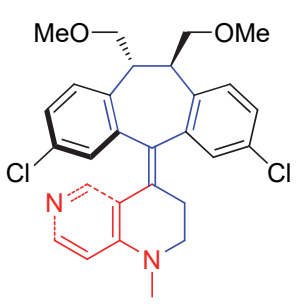

State I ( $R$-selectivity)
$(10 R, 11 R, P)-8$

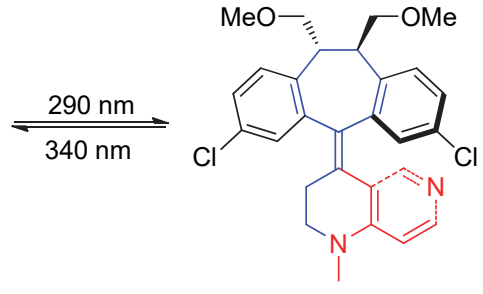

$(10 R, 11 R, M)-8$

State II (S-selectivity)

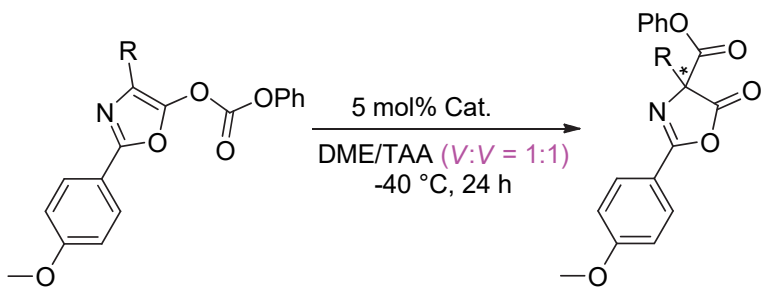

State I: $R$-form, up to $91 \%$ ee, up to $86 \%$ yield

State II: $S$-form, up to $94 \%$ ee, up to $83 \%$ yield

图式 $8 \mathrm{Chen}$ 和 $\mathrm{Yu}$ 等发展的基于螺烯的光驱动手性开关催化 剂及其催化的可切换不对称 Steglich 重排反应

Scheme 8 A switchable chiral helicene catalyst and its application in switchable asymmetric Steglich rearrangement reactions developed by Chen and Yu et al.

\section{2 金属配位驱动的手性开关催化体系}

金属配位键已被广泛应用于超分子组装体的构 建 ${ }^{[27]}$, 通过在金属配位体系中引入 hemilabile 配体、外 加其他金属或 $\mathrm{CO}$ 、腈、异腈、吡啶、二胺及卤素阴离 子等辅助配体, 可以实现金属配位的动态调控, 从而对 金属催化反应产生影响 ${ }^{[28]}$.

Mirkin 小组 ${ }^{[28]}$ 在金属配位驱动的变构催化剂 (allosteric catalyst)领域开展了系列研究工作, 他们发展 了 WLA (The Weak-Link Approach) 和 HILR (halide-induced ligand rearrangement)两种新策略, 成功构筑 了一系列非手性大环金属配合物以及三明治式的三层 变构催化剂体系 ${ }^{[29-31]}$, 实现了通过配位相互作用调控催 化活性位点之间的相对距离. 2003 年, 他们 ${ }^{[32]}$ 报道了第 一例基于超分子配位化学的手性变构催化剂(Scheme 9), 通过在手性 salen-Cr(III)催化活性中心远端引入两个 hemilabile $P, S$-配体, 使其与 Rh(I)配位(形成结构控制位 点)合成了四金属中心大环配合物 9a. Rh(I)金属中心与 $\mathrm{CO}$ 和 $\mathrm{Cl}^{-}$的可逆结合(形成 State II), 会改变整个大环催 化剂的形貌及两个 salen-Cr(III)催化活性中心的相对距 离, 进而在他们催化的 $\mathrm{TMSN}_{3}$ 对 1,2-环氧环己烷的不对 称开环反应中, 这两种不同结构的催化剂表现出不同的 催化活性. State I 催化反应的速率是 salen-Cr(III)单体催 化剂的 20 倍, 而 Satet II 催化反应的速率是 State I 的 2 倍, 且 Satet II 催化反应能够获得 $68 \%$ ee 的手性产物,
远远高于手性 salen-Cr(III)单体催化剂给出的 $12 \%$ 的 $e e$ 值. 这一催化结果符合 salen-Cr(III)催化中双金属协同 催化的机理 ${ }^{[33]}$.

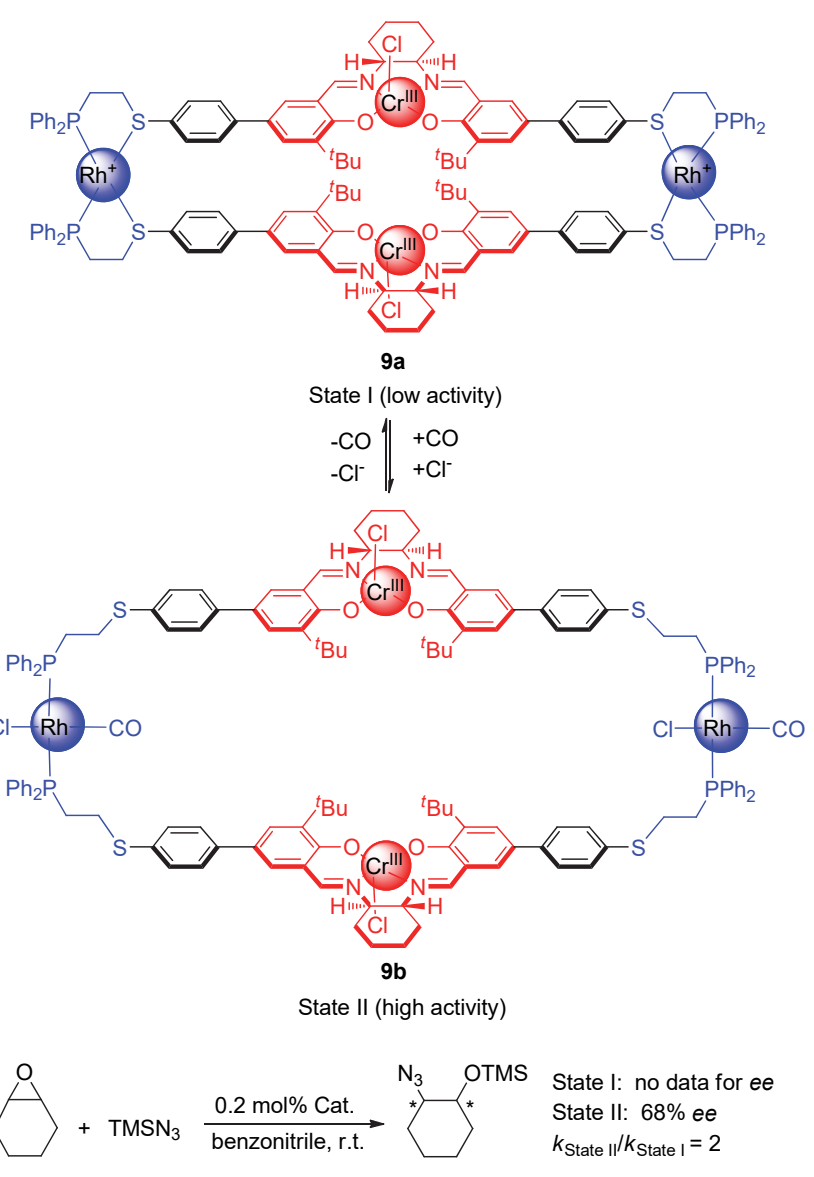

图式 9 Mirkin 小组发展的大环变构催化剂及其催化的可切 换不对称开环反应

Scheme 9 A macrocyclic allosteric catalyst and its application in switchable asymmetric ring-opening reactions developed by Mirkin group

随后, Mirkin 小组 ${ }^{[34]}$ 对上述大环变构金属催化剂进 行改造，去掉一个铑结构控制位点，制备出了新型双 salen-Cr(III)手性 “分子钳” 催化剂(Scheme 10). 该催化 剂同样可以通过 $\mathrm{CO}$ 和 $\mathrm{Cl}^{-}$来可逆调控 State I 和 State II 的形成，同时解决了大环金属催化剂溶解度低的问题. 在 $\mathrm{TMSN}_{3}$ 对 1,2-环氧环已烷的不对称开环反应中, 采用 高浓度 $\left(7.2 \times 10^{-3} \mathrm{~mol} / \mathrm{L}\right)$ 催化剂时, State I 给出 $80 \% \mathrm{ee}$ 的产物, State II 给出 $74 \%$ 的 $e e$ 值; 而当催化剂浓度降低 为 $0.14 \times 10^{-3} \mathrm{~mol} / \mathrm{L}$ 时, State I 和 State II 分别给出 $49 \%$ 和 $21 \%$ 的 $e e$ 值, 表现出分子内协同催化的差异 ${ }^{[33]}$. 随 后, 他们利用 $\mathrm{CO}$ 气体作为变构试剂, 通过通入和去除 $\mathrm{CO}$, 实现了不对称催化反应的原位可逆调控. 这是第 一例可以对催化活性和选择性进行原位调控的人工变 构催化剂. 


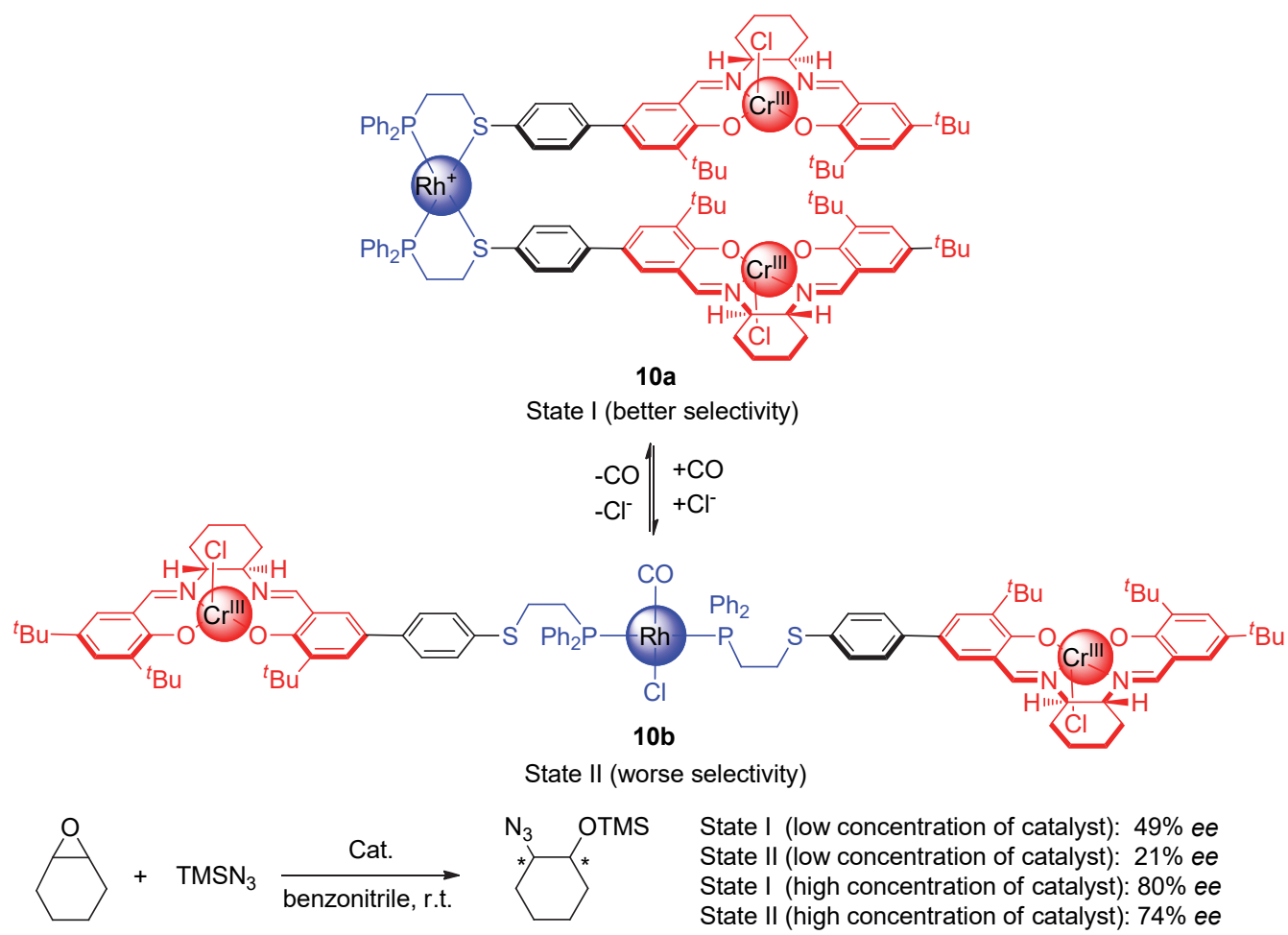

图式 10 Mirkin 小组发展的钳形分子催化剂及其催化的可切换不对称开环反应

Scheme 10 Catalytic molecular tweezers and their application in switchable asymmetric ring-opening reactions developed by Mirkin group

2015 年, 范青华小组 ${ }^{[35]}$ 设计合成了基于单氮杂冠 醚的手性亚磷酰胺单齿配体, 发展了金属阳离子驱动的 手性铑催化剂. 在 $\mathrm{Rh}(\mathrm{I})$ 催化脱氢氨基酸酯的不对称氢 化反应中, 通过添加和箱取碱金属离子, 以 $>100$ 的高 开/关比(State II/State I) 实现了催化反应 “开” 与 “关” 的高效和可逆转换(Scheme 11). 在未添加钠离子的情况 下, 氮杂冠醚中的富电子氧原子可以与配位不饱和的 $\mathrm{Rh}(\mathrm{I})$ 离子配位 (State I), 催化活性中心被关闭, 底物的 转化率小于 $1 \%$; 当加入 2 equiv.的 NaBArF 催化剂后, 氮杂冠醚选择性地与 $\mathrm{Na}^{+}$识别, 形成更加稳定的主-客 体复合物, 进而与 $\mathrm{Rh}(\mathrm{I})$ 金属中心解离(State II), 使催化 剂恢复活性, 在温和的反应条件下以优异的对映选择性 实现了一系列氢化反应的完全转化. 随后, 加入穴醚䁴 取氮杂冠醚中的 $\mathrm{Na}^{+}$, 氮杂冠醚重新与 $\mathrm{Rh}(\mathrm{I})$ 金属配位, 催化剂重新回到失活状态. 这是首例过渡金属催化的刺 激响应性可开关不对称反应的报道, 同时, 这一研究成 果为发展新型智能超分子金属催化体系提供了新的思 路和途径.

\section{$3 \mathrm{pH}$ 响应的手性开关催化体系}

利用输入性化学信号改变体系的 $\mathrm{pH}$ 值来驱动/阻止 化学反应是自然界中非常重要的一种调控方式 ${ }^{[1 b]}$. 近 年来, $\mathrm{pH}$ 刺激响应的人工分子机器已取得了长足的发 展 ${ }^{[36]}$, 并已被应用于超分子催化研究领域 ${ }^{[5 \mathrm{~d}, 37]}$. 然而,
以 $\mathrm{pH}$ 作为化学刺激信号的手性催化剂体系的研究仍处 于起步阶段.

Leigh 小组在这一领域作出了杰出贡献, 取得了具 有影响力的系列研究成果. 2012 年, 他们利用冠醚与二 芐胺盐轮烷对酸/碱的响应性能, 通过调控反应体系的 $\mathrm{pH}$ 值, 调节二芐胺催化活性中心在裸露和包裹状态间 可逆转换, 发展了开关可控的非手性超分子有机催化 剂 ${ }^{[38]}$. 随后, 他们将手性仲胺引入轮烷结构 (Scheme 12), 在酸碱调控下, 利用 State I 与 State II 的可逆转换, 实现了 1,3 -二苯基- 1,3 -丙二酮对 $\alpha, \beta$-不饱和醛的可切换 不对称 Michael 加成反应 ${ }^{[39]}$. 在酸性条件下, 冠醚与仲 胺盐位点结合, 催化剂 12a 没有活性; 在碱性条件下, 冠醚与轴上三氮唑盐位点结合, 手性仲胺催化活性位点 得以释放, 12b 能以最高 $70 \%$ 的收率和 $88 \%$ 的对映体过 量给出手性二酮加成产物. 他们同时实现了这一不对称 催化反应的原位可逆开关调控.

最近, Leigh 等基于他们发展的机械点手性轮烷的 设计理念 ${ }^{[40]}$, 设计合成了一种新型刺激响应性手性轮

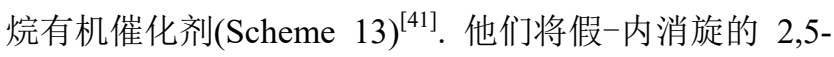
二取代吡咯催化中心引入轴的设计, 在其两侧分别通过 酰胺键连接吡啶基酰基腙和甘氨酰胺官能团及大位阻 封端基团, 通过光照/酸动态可逆调控芐基酰胺大环与 轴不同位置的识别与组装(State I 和 State II), 从而打破 吡咯催化中心的局部对称性, 使其具备了相反的手性环 


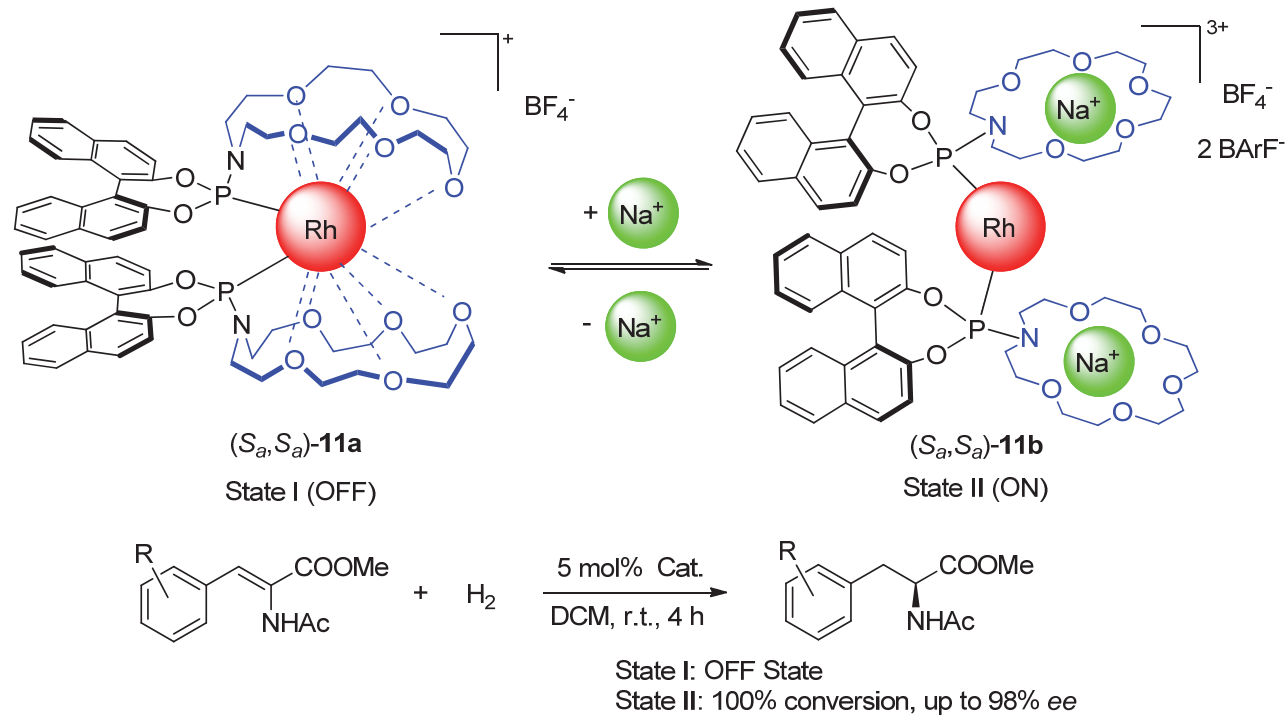

图式 11 Fan 小组发展的金属阳离子驱动的手性铑催化剂及其催化的可切换不对称氢化反应

Scheme 11 A cation-responsive chiral Rh-catalyst and its application in switchable asymmetric hydrogenation developed by Fan group

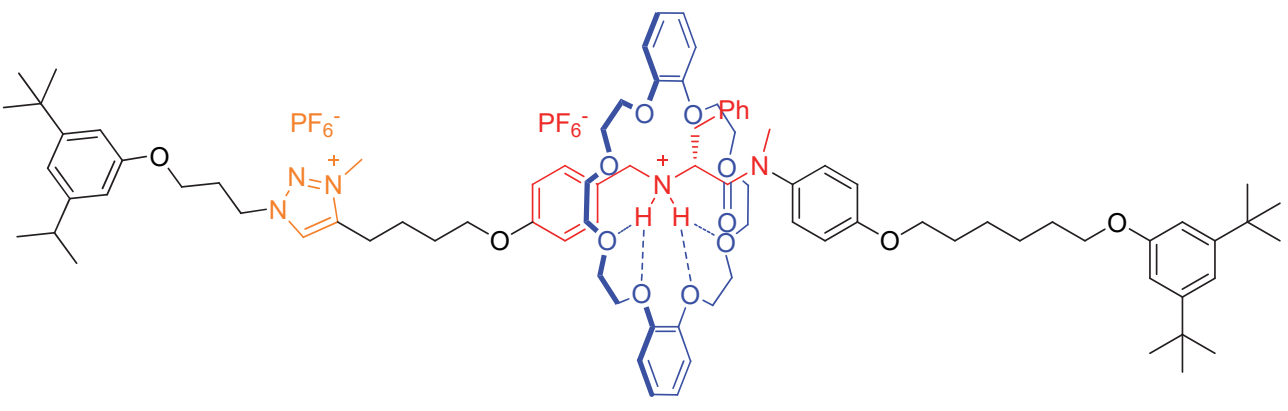

$12 \mathrm{a}$

State I (OFF)

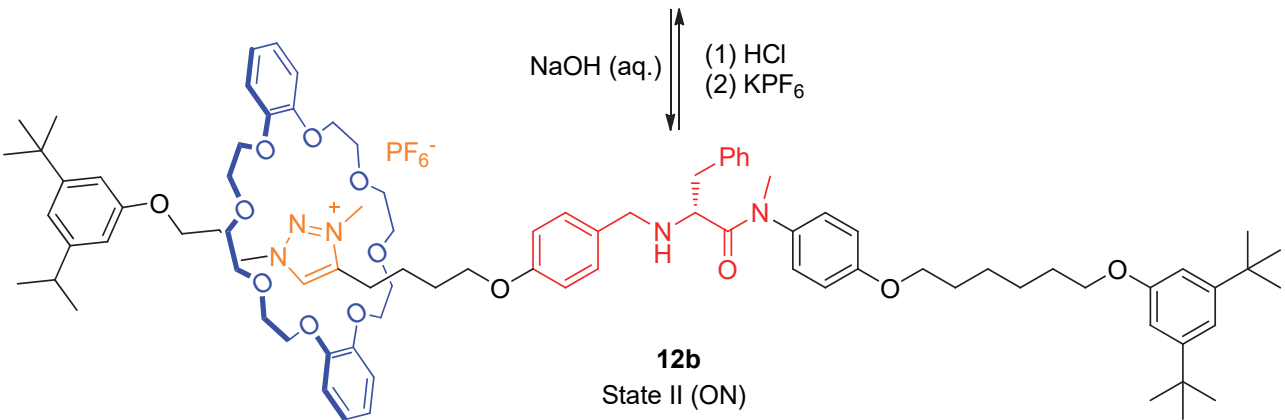

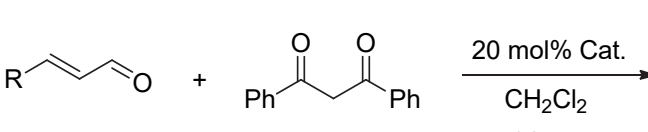

$$
\begin{aligned}
& 10{ }^{\circ} \mathrm{C}, 48 \mathrm{~h} \\
& \mathrm{R}=\mathrm{Me}, \mathrm{Et}, n-\mathrm{Pr}
\end{aligned}
$$

图式 12 Leigh 小组发展的手性轮烷开关催化剂及其催化的可切换不对称 Michael 加成反应

Scheme 12 A switchable chiral rotaxane catalyst and its application in switchable asymmetric Michael addition reactions developed by Leigh group

境. 在己醛对乙烯基二砜的共轭加成反应中, $E-13$ 催化 剂和 $Z-13$ 催化剂给出绝对构型相反的产物, 且 $E-13$ (给
出 $40 \%$ ee 的手性产物)的手性诱导能力略高于 $Z-13$ (20\% ee 的产物). 虽然 $E-13$ 和 $Z-13$ 催化反应的对映选 


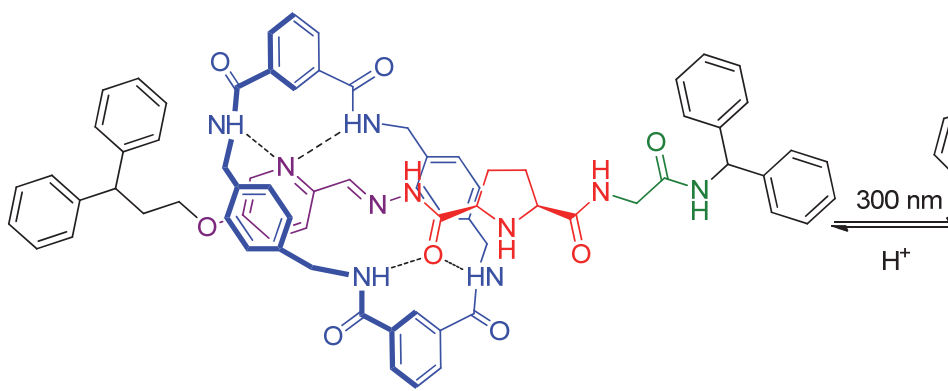

E-13

State I (S-selctivity)

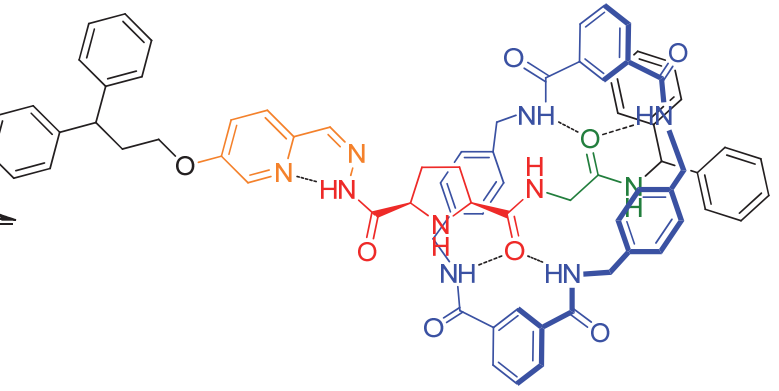

Z-13

State II (R-selctivity)<smiles>C=C([CH+]CCC(=O)c1ccccc1)S(=O)(=O)c1ccccc1</smiles>

(1) $10 \mathrm{~mol} \%$ Cat.
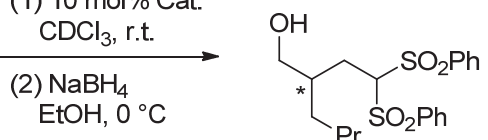

State I: S-form, $40 \%$ ee State II: $R$-form, $20 \%$ ee

图式 13 Leigh 小组发展的手性轮烷有机催化剂及其催化的对映选择性发散合成

Scheme 13 A rotaxane-based chiral organocatalyst developed by Leigh group and its application for enantiodivergent synthesis

择性不高, 但明显高于轴做催化剂的反应结果. 这是第 一例刺激响应轮烷成功实现对映选择性发散催化 (enantiodivergent catalysis)的报道.

除了在刺激响应性手性轮烷催化剂方面的探索, Leigh 小组 ${ }^{[42]}$ 还开发了可开关的双功能手性金鸡纳碱方酰胺催化剂(Scheme 14). 他们 ${ }^{[43]}$ 以吡啶基酰基腙分 子开关作为调控模块, 分别引入手性方酰胺催化单元和 氢键受体硝基苯催化活性抑制基团. 当分子开关处于反 式(E-14)时, 硝基苯基元远离催化活性中心, 并释放出 催化活性中心(ON State); 相反, 分子开关处于顺式 (Z-14)时, 硝基苯基元接近催化活性中心并形成分子内 氢键复合物, 从而抑制催化活性(OFF State); 而且通过 光/酸(加热)可实现对催化剂开与关状态的可逆调控 (State I 和 State II). 同时, 作者通过巧妙地改变催化剂 与分子开关的连接位置, 制备了另一手性构型的金鸡纳 碱一方酰胺开关催化剂( $Z-15$ 和 $E-15)$, 但其开关状态正 好与催化剂 14 相反. 随后, 作者选择一系列共轭加成反 应来评价催化剂的催化性能, 在 “开” 的状态下, E-14 可以最高 $95 \%$ 的转化率和 $90 \%$ ee 得到手性产物, Z-15 得到最高 $95 \%$ 的转化率和 $86 \%$ ee 绝对构型相反的手性 产物, 其催化效果与已报道的双功能有机催化剂相謧 美. 另一方面, 作者将两个催化剂相混合, 期望在外界 刺激下, 交替制备绝对构型相反的手性产物. 遗憾的是, 该混合催化剂不能催化反应的发生, 可能是因为两种催 化剂形成聚集体而失活.

\section{4 氧化还原驱动的手性开关催化体系}

氧化还原驱动的人工开关催化体系已有很多报 道 ${ }^{[44]}$, 其中大多数局限于对催化剂活性的调控, 主要采 取以下两种设计理念 ${ }^{[5 b]}$ : 第一, 使用包含氧化还原活性 官能团(二茂铁或二茂钴)的配体, 通过改变配体中金属
的价态, 进而对催化中心的电性和位阻产生影响; 第二, 通过氧化还原直接改变催化中心金属的价态，进而实现 对其催化性质的调控.

2000 年, Canary 等 ${ }^{[45]}$ 发现连有两个喹啉基团的 $L-$ 蛋氨酸衍生物可与不同价态的铜进行配位，铜的氧化态 决定了配合物的螺旋手性, 同时实现了氧化还原驱动的 铜配合物螺旋手性的可逆翻转. 2012 年, 基于这一手性 铜配合物的基本骨架, 通过在 $L$-蛋氨醇配体远端引入两 个具有催化活性的非手性脲官能团, 他们 ${ }^{[46]}$ 设计合成 了首例氧化还原驱动的手性催化剂(Scheme 15), 通过 中心金属价态的改变能够调控配体的配位方式(State I 和 State II), 从而使金属中心处于不同的手性环境. 在 催化丙二酸二乙酯对 trans- $\beta$-硝基苯乙烯的加成反应中, 使用 $\Delta-17$ (State I) 可得到 $S$ 构型产物(产率 55\%, 72\% ee), $\Lambda$-17 (State II)则给出 $R$ 构型产物(产率 $40 \%, 70 \% e e$ ). 同 时, $\Delta-17$ 可以原位被抗坏血酸还原得到 $\Lambda-17$, 其催化结 果与单独制备的催化剂类似，以 $43 \%$ 的产率和 $71 \%$ 的对 映体过量得到 $R$ 构型产物. 随后，他们 ${ }^{[47]}$ 对该催化剂进 行了更深入的研究, 进一步拓展了底物范围, 当以不对 称的丙二酸酯作为反应原料时, 通过氧化还原调控, 同 样能够实现产物对映选择性的翻转.

2015 年, 崔秀灵、罗三中和吴养洁等 ${ }^{[48]}$ 首次将具有 氧化还原活性的二茂铁基团与手性伯胺相结合，报道了 氧化还原驱动有机小分子催化芳香醛与烷基酮的直接 不对称 aldol 反应(Scheme 16). 在对硝基苯甲醛和环己 酮的反应中, 当使用含 $\mathrm{Fe}(\mathrm{II})$ 的 $\mathbf{1 8 a}$ (State I) 为催化剂时, 仅以 $38 \%$ 的产率和较差的立体选择性( anti/syn $=42: 58$, $24 \% e e)$ 得到绝对构型为 $(1 R, 2 S)$ 的手性产物; 体系中加 入氧化剂 $\left[\mathrm{FeCp}_{2}\right]^{+} \mathrm{BF}_{4}^{-}$后, 催化剂二茂铁基团中的 $\mathrm{Fe}(\mathrm{II})$ 被氧化为 $\mathrm{Fe}(\mathrm{III})$, 该二茂铁离子作为路易斯酸既 活化芳香醛底物, 同时又有利于产物的立体选择性控 


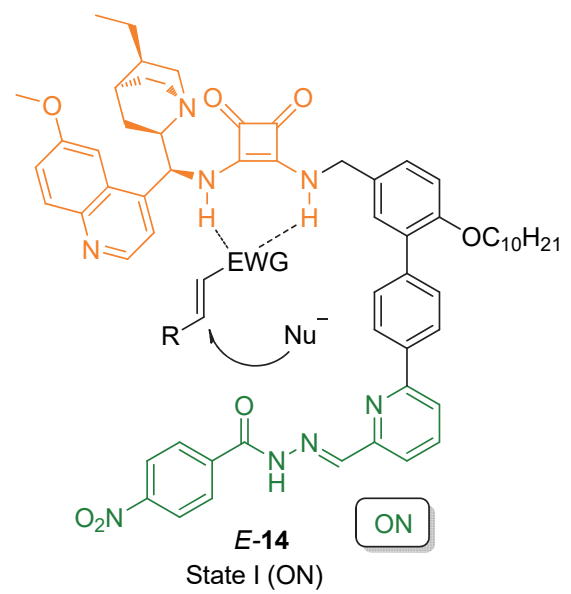

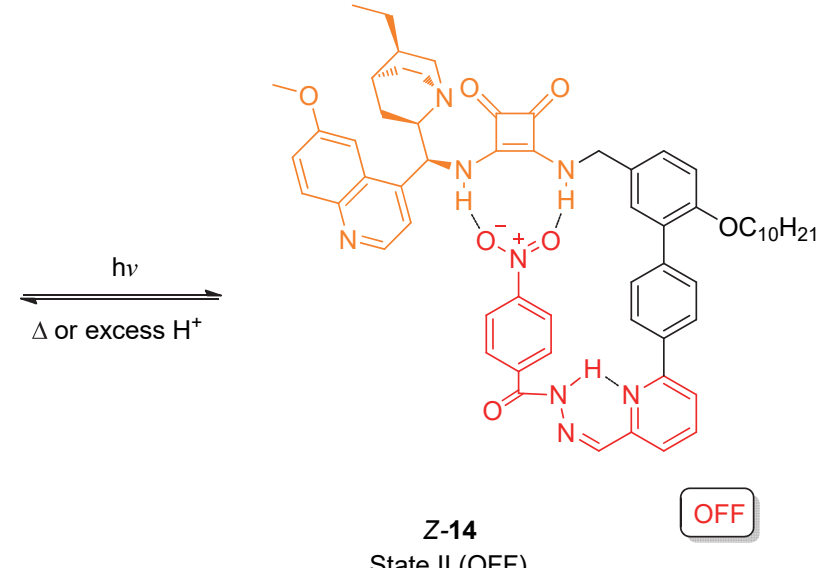

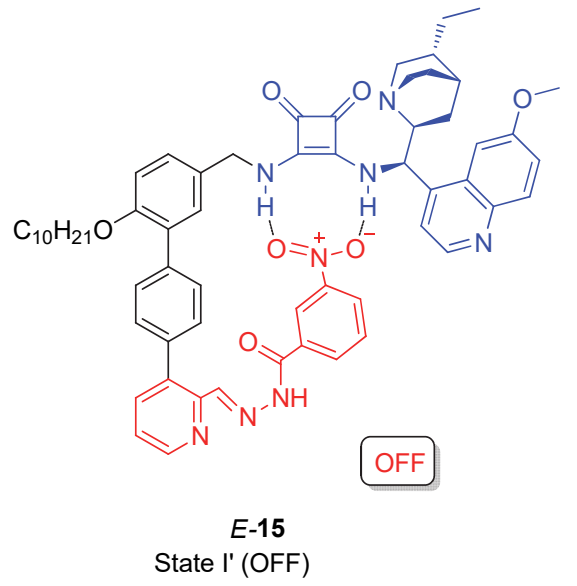
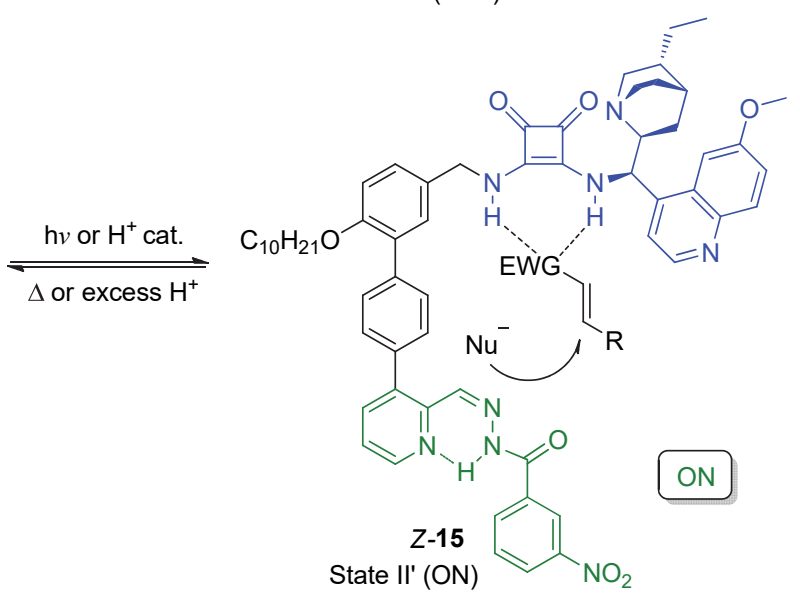

$$
\underset{\text { State II and State I': OFF State }}{\longrightarrow}
$$

图式 14 Leigh 小组发展的可开关双功能手性有机催化剂及其催化的可切换不对称催化反应

Scheme 14 Chiral switchable bifunctional organocatalysts and its application in switchable asymmetric reactions developed by Leigh group

制，因此催化剂 18b (State II)的反应活性和立体选择性 都得到大幅度提升, 以 $94 \%$ 的产率、 $90 ： 10$ 的顺一反比 和 $93 \%$ 的 $e e$ 值得到相同的手性产物. 有趣的是, 由于 $18 \mathrm{a}$ 与 $\left[\mathrm{FeCp}_{2}\right]^{+} \mathrm{BF}_{4}^{-}$原位生成的离子型催化剂 $18 \mathrm{~b}$ 可以 用沉淀法从体系中析出, 他们进一步实现了催化剂的回 收和重复使用.

\section{5 总结与展望}

综上所述，受酶变构催化的启发，人工刺激响应性 手性催化体系的研究在过去 20 年间取得了一些重要进 展. 通过将不同类型刺激响应性官能团引入手性催化剂 的设计, 实现了基于光/热驱动、配位驱动、 $\mathrm{pH}$ 驱动以 及氧化还原驱动的多类可切换不对称催化反应. 其中代 表性的催化体系包括 Feringa 小组研制的基于光/热驱动
分子马达的手性开关催化剂、Mirkin 小组发展的金属配 位驱动的手性双 salen-Cr(III)开关催化剂以及 Leigh 小组 发展的 $\mathrm{pH}$ 响应的手性轮烷开关催化剂.

虽然刺激响应性可切换不对称催化这一新兴领域发展 迅速，但目前仍然存在许多不足，同时也面临诸多挑战. 首先，该领域的研究工作还处于起步阶段，目前主要局 限于概念性研究, 刺激响应模式相对简单, 不对称催化 反应类型也十分有限; 其次, 刺激响应模块和催化活性 位点的兼容性不足，刺激响应模块的引入对催化反应的 活性和立体选择性造成一定的负面影响, 从而使得在催 化剂不同状态下反应速率的开/关比或立体选择性差异 不大; 此外, 目前还没能实现通过刺激响应性手性催化 剂的组合进行多组份、多个平行反应的精准时空控制, 无法完成不对称串联反应或控制多种手性产物的分级 


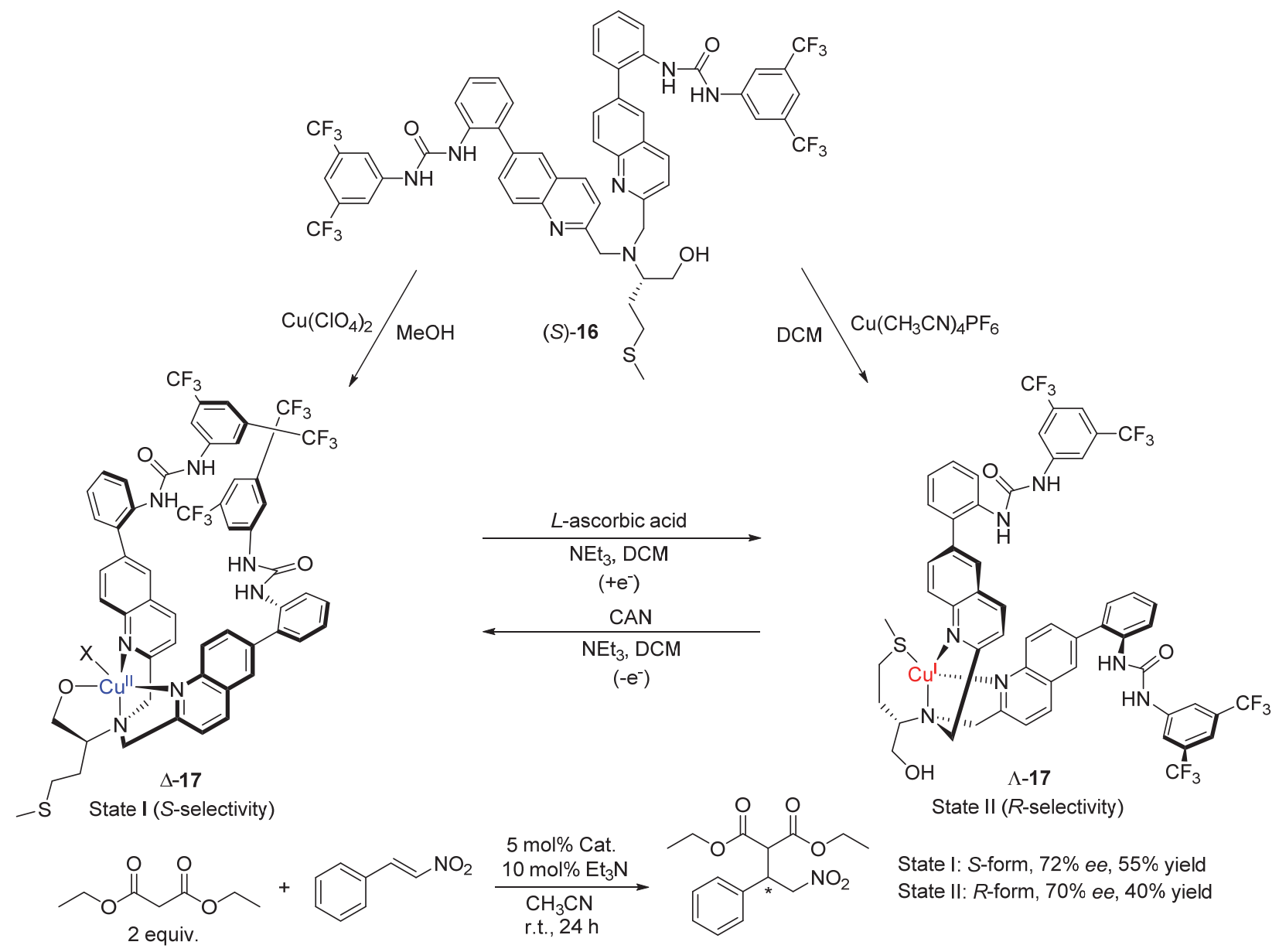

图式 15 Canary 小组发展的氧化还原驱动的手性开关催化剂及其催化的可切换不对称催化反应

Scheme 15 A redox-switchable chiral catalyst and its application in switchable asymmetric reactions developed by Canary group

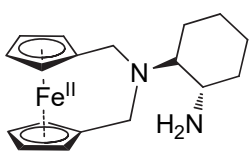

$18 a$

State I (low activity and selectivity)<smiles>O=C1CCCCC1</smiles>

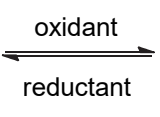<smiles>O=Cc1ccc([N+](=O)[O-])cc1</smiles>

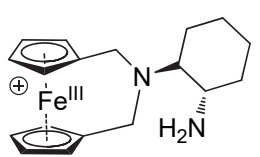

$18 b$

State II (high activity and selectivity)

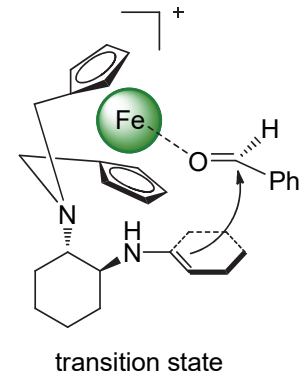

State I: $42: 58$ antilsyn, $24 \%$ ee, $38 \%$ yield State II: $90: 10$ anti/syn, $93 \%$ ee, $94 \%$ yield

图式 16 Cui, Luo 和 $\mathrm{Wu}$ 等发展的氧化-还原驱动的手性开关有机催化剂及其催化的可切换不对称 aldol 反应

Scheme 16 A redox-responsive chiral organocatalyst and its application in switchable asymmetric aldol reactions developed by Cui, Luo and $\mathrm{Wu}$ et al.

输出.

最近, Leigh 小组 ${ }^{[49]}$ 设计合成了一种可编程分子机 器手性催化剂(Scheme 17), 经烯烃复分解反应上载 $\alpha, \beta$ 不饱和醛底物后, 通过编程式的操作和 $\mathrm{pH}$ 调控, 实现 了 “一锅法” 有机胺催化的亲核/亲电加成串联反应以及
产物的还原和水解等多个反应过程, 实现了分子机器驱 动的立体发散式不对称合成，分别得到了全部四个立体 异构体. 虽然产物的非对映选择性和对映选择性均不是 很高, 但显示了人工刺激响应性手性开关催化剂的未来 发展潜力. 相信随着不对称催化和超分子化学的不断发 


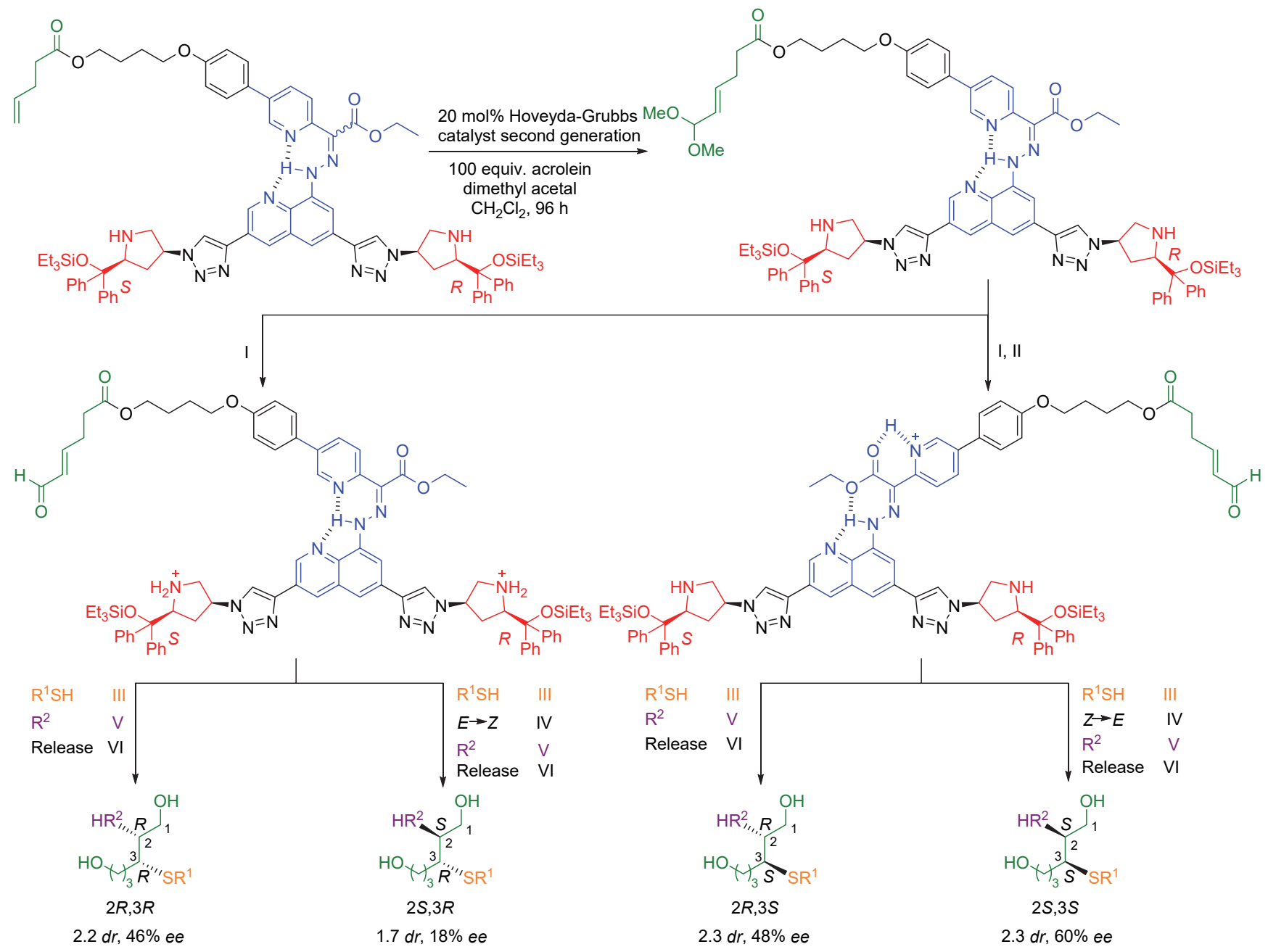

Reagents and conditions: (I) $\mathrm{CF}_{3} \mathrm{COOH}$ (2.2 equiv.), r.t., $10 \mathrm{~min}, \mathrm{CD}_{2} \mathrm{Cl}_{2}(1 \mathrm{mmol} / \mathrm{L})$; (II) $(E \rightarrow Z), \mathrm{CF}_{3} \mathrm{COOH}\left(3.0\right.$ equiv.), r.t., $5 \mathrm{~h}, \mathrm{CD}_{2} \mathrm{Cl}_{2}(1$ mmol/L); (III) $\mathrm{R}^{1} \mathrm{SH}$ (100 equiv.), $0{ }^{\circ} \mathrm{C}, 30 \sim 41 \mathrm{~h}, \mathrm{CD}_{2} \mathrm{Cl}_{2}(1 \mathrm{mmol} / \mathrm{L}) ;(\mathrm{IV})(E \rightarrow Z), \mathrm{CF}_{3} \mathrm{COOH}\left(6.0\right.$ equiv.), $0{ }^{\circ} \mathrm{C}, 20 \mathrm{~h}, \mathrm{CD}_{2} \mathrm{Cl}_{2}(1 \mathrm{mmol} / \mathrm{L})$; (IV) $(Z \rightarrow E), \mathrm{Et}_{3} \mathrm{~N}$ (7.0 equiv.), $120 \mathrm{~mL} \mathrm{CD}{ }_{3} \mathrm{CN}, 0{ }^{\circ} \mathrm{C}, 6 \mathrm{~h}, \mathrm{CD}_{2} \mathrm{Cl}_{2}(1 \mathrm{mmol} / \mathrm{L}) ;(\mathrm{V}) \mathrm{R}^{2}$ (200 equiv.), $0{ }^{\circ} \mathrm{C}, 24 \mathrm{~h}, \mathrm{CD}_{2} \mathrm{Cl}_{2}(1 \mathrm{mmol} / \mathrm{L}) ;(\mathrm{VI}) \mathrm{NaBH}_{4}$, $\mathrm{MeOH}, 0{ }^{\circ} \mathrm{C}, 2 \mathrm{~h}, \mathrm{CD}_{2} \mathrm{Cl}_{2}(1 \mathrm{mmol} / \mathrm{L})$, then $\mathrm{LiAlH}_{4}, \mathrm{THF},-78{ }^{\circ} \mathrm{C}$ to r.t., 1 h. $\mathrm{R}^{1}=\left(\mathrm{CH}_{2}\right)_{2}\left(\mathrm{CF}_{2}\right)_{7} \mathrm{CF}_{3}, \mathrm{R}^{2}=\mathrm{H}_{2} \mathrm{C}=\mathrm{C}\left(\mathrm{SO}_{2} \mathrm{Ph}\right)_{2}$.

图式 17 Leigh 小组发展的可编程手性分子机器催化剂及其催化的立体发散性不对称合成

Scheme 17 A programmable chiral molecular machine catalyst and its application in stereodivergent asymmetric synthesis developed by Leigh group

展，二者的相互融合 ${ }^{[50]}$ 会给刺激响应性可切换不对称 催化带来更多新的灵感和创意，化学家们将会设计出更 加高效和精致的智能手性催化体系, 实现更加高效、高 选择性的复杂仿生不对称催化网络.

\section{References}

[1] (a) Traut, T. Enzyme Activity: Allosteric Regulation, John Wiley \& Sons Ltd, Chichester, 2014.

(b) Traut, T. Allosteric Regulatory Enzymes, Springer, New York, 2008.

[2] van Leeuwen, P. W. N. M. Homogeneous Catalysis: Understanding the Art, Springer, Dordrecht, 2004.

[3] van Leeuwen, P. W. N. M. Supramolecular Catalysis, Wiley-VCH, Weinheim, 2008.

[4] (a) Raynal, M.; Ballester, P.; Vidal-Ferran, A.; van Leeuwen, P. W. N. M. Chem. Soc. Rev. 2014, 43, 1660.

(b) Raynal, M.; Ballester, P.; Vidal-Ferran, A.; van Leeuwen, P. W.
N. M. Chem. Soc. Rev. 2014, 43, 1734.

(c) Wang, Q.-Q. In Handbook of Macrocyclic Supramolecular Assembly, Eds.: Liu, Y.; Chen, Y.; Zhang, H.-Y., Springer, Singapore, 2019, pp. 1 124.

(d) Tang, Y.; He, Y.; Feng, Y.; Fan. Q. Prog. Chem. 2018, 30, 476 (in Chinese).

(唐雨平, 何艳梅, 冯宇, 范青华, 化学进展, 2018, 30, 476.)

[5] (a) Lüning, U. Angew. Chem., Int. Ed. 2012, 51, 8163.

(b) Blanco, V.; Leigh, D. A.; Marcos, V. Chem. Soc. Rev. 2015, 44, 5341.

(c) Vlatković, M.; Collins, B. S. L.; Feringa, B. L. Chem.-Eur. J. 2016, 22, 17080.

(d) van Dijk, L.; Tilby, M. J.; Szpera, R.; Smith, O. A.; Bunce, H. A. P.; Fletcher, S. P. Nat. Rev. Chem. 2018, 2, 0117.

[6] (a) Cao, W.; Feng, X.; Liu, X. Org. Biomol. Chem. 2019, 17, 6538. (b) Ding, Z.-Y.; Chen, F.; Qin, J.; He, Y.-M.; Fan, Q.-H. Angew. Chem., Int. Ed. 2012, 51, 5706.

[7] Kasprzyk-Hordern, B. Chem. Soc. Rev. 2010, 39, 4466.

[8] Romanazzi, G.; Degennaro, L.; Mastrorilli, P.; Luisi, R. ACS Catal. 2017, 7, 4100. 
[9] (a) Yamamoto, T.; Yamada, T.; Nagata, Y.; Suginome, M. J. Am. Chem. Soc. 2010, 132, 7899.

(b) Akai, Y.; Yamamoto, T.; Nagata, Y.; Ohmura, T.; Suginome, M. J. Am. Chem. Soc. 2012, 134, 11092.

(c) Nagata, Y.; Nishikawa, T.; Suginome, M. J. Am. Chem. Soc. 2014, 136, 15901

(d) Akai, Y.; Konnert, L.; Yamamoto, T.; Suginome, M. Chem. Commun. 2015, 51, 7211 .

(e) Ke, Y.-Z.; Nagata, Y.; Yamada, T.; Suginome, M. Angew. Chem., Int. Ed. 2015, 54, 9333.

[10] Stoll, R. S.; Hecht, S. Angew. Chem., Int. Ed. 2010, 49, 5054.

[11] Irie, M. Chem. Rev. 2000, 100, 1685.

[12] Sud, D.; Norsten, T. B.; Branda, N. R. Angew. Chem., Int. Ed. 2005, 44, 2019.

[13] (a) Koumura, N.; Zijlstra, R. W. J.; van Delden, R. A.; Harada, N.; Feringa, B. L. Nature 1999, 401, 152.

(b) Eelkema, R.; Pollard, M. M.; Vicario, J.; Katsonis, N.; Ramon, B. S.; Bastiaansen, C. W. M.; Broer, D. J.; Feringa, B. L. Nature 2006, 440, 163.

[14] Dorel, R.; Feringa, B. L. Chem. Commun. 2019, 55, 6477.

[15] Wang, J.; Feringa, B. L. Science 2011, 331, 1429.

[16] Vlatković, M.; Bernardi, L.; Otten, E.; Feringa, B. L. Chem. Commun. 2014, 50, 7773.

[17] Trost, B. M.; Van Vranken, D. L.; Bingel, C. J. Am. Chem. Soc. $1992,114,9327$.

[18] Zhao, D.; Neubauer, T. M.; Feringa, B. L. Nat. Commun. 2015, 6, 6652 .

[19] (a) Juwarker, H.; Lenhardt, J. M.; Pham, D. M.; Craig, S. L. Angew. Chem., Int. Ed. 2008, 47, 3740.

(b) Juwarker, H.; Jeong, K.-S. Chem. Soc. Rev. 2010, 39, 3664.

[20] Dorel, R.; Feringa, B. L. Angew. Chem., Int. Ed. 2020, 59, 785.

[21] (a) Koumura, N.; Geertsema, E. M.; van Gelder, M. B.; Meetsma, A.; Feringa, B. L. J. Am. Chem. Soc. 2002, 124, 5037.

(b) Vicario, J.; Walko, M.; Meetsma, A.; Feringa, B. L. J. Am. Chem. Soc. 2006, 128, 5127.

(c) Klok, M.; Walko, M.; Geertsema, E. M.; Ruangsupapichat, N.; Kistemaker, J. C. M.; Meetsma, A.; Feringa, B. L. Chem.-Eur. J. 2008, 14, 11183.

[22] Pizzolato, S. F.; Collins, B. S. L.; van Leeuwen, T.; Feringa, B. L. Chem.-Eur. J. 2017, 23, 6174.

[23] Pizzolato, S. F.; Štacko, P.; Kistemaker, J. C. M.; van Leeuwen, T.; Otten, E.; Feringa, B. L. J. Am. Chem. Soc. 2018, 140, 17278.

[24] Pizzolato, S. F.; Štacko, P.; Kistemaker, J. C. M.; van Leeuwen, T.; Feringa, B. L. Nat. Catal. 2020, 3, 488.

[25] (a) Chen, C.-T.; Chou, Y.-C. J. Am. Chem. Soc. 2000, 122, 7662. (b) Chen, W.-C.; Lee, Y.-W.; Chen, C.-T. Org. Lett. 2010, 12, 1472. (c) Chen, C.-T.; Chen, C.-H.; Ong, T.-G. J. Am. Chem. Soc. 2013, $135,5294$.

[26] Chen, C.-T.; Tsai, C.-C.; Tsou, P.-K.; Huang, G.-T.; Yu, C.-H. Chem. Sci. 2017, 8, 524.

[27] (a) Holliday, B. J.; Mirkin, C. A. Angew. Chem., Int. Ed. 2001, 40, 2022.

(b) Caulder, D. L.; Raymond, K. N. Acc. Chem. Res. 1999, 32, 975.

[28] Oliveri, C. G.; Ulmann, P. A.; Wiester, M. J.; Mirkin, C. A. Acc. Chem. Res. 2008, 41, 1618.

[29] Wiester, M. J.; Ulmann, P. A.; Mirkin, C. A. Angew. Chem., Int. Ed. 2011, 50, 114
[30] Gianneschi, N. C.; Masar III, M. S.; Mirkin, C. A. Acc. Chem. Res. 2005, 38, 825 .

[31] Yoon, H. J.; Kuwabara, J.; Kim, J.-H.; Mirkin, C. A. Science 2010, 330,66 .

[32] Gianneschi, N. C.; Bertin, P. A.; Nguyen, S. T.; Mirkin, C. A.; Zakharov, L. N.; Rheingold, A. L. J. Am. Chem. Soc. 2003, 125, 10508.

[33] Hansen, K. B.; Leighton, J. L.; Jacobsen, E. N. J. Am. Chem. Soc. 1996, 118, 10924.

[34] Gianneschi, N. C.; Cho, S.-H.; Nguyen, S. T.; Mirkin, C. A. Angew Chem., Int. Ed. 2004, 43, 5503.

[35] Ouyang, G.-H.; He, Y.-M.; Li, Y.; Xiang, J.-F.; Fan, Q.-H. Angew. Chem., Int. Ed. 2015, 54, 4334.

[36] (a) Coskun, A.; Banaszak, M.; Astumian, R. D.; Stoddart, J. F.; Grzybowski, B. A. Chem. Soc. Rev. 2012, 41, 19.

(b) Zhang, L.; Marcos, V.; Leigh, D. A. Proc. Natl. Acad. Sci. U. S. A. 2018, 115, 9397.

[37] Leigh, D. A.; Marcos, V.; Wilson, M. R. ACS Catal. 2014, 4, 4490.

[38] Blanco, V.; Carlone, A.; Hänni, K. D.; Leigh, D. A.; Lewandowski, B. Angew. Chem., Int. Ed. 2012, 51, 5166.

[39] Blanco, V.; Leigh, D. A.; Marcos, V.; Morales-Serna, J. A.; Nussbaumer, A. L. J. Am. Chem. Soc. 2014, 136, 4905.

[40] (a) Alvarez-Pérez, M.; Goldup, S. M.; Leigh, D. A.; Slawin, A. M. Z. J. Am. Chem. Soc. 2008, 130, 1836.

(b) Cakmak, Y.; Erbas-Cakmak, S.; Leigh, D. A. J. Am. Chem. Soc. 2016, 138, 1749 .

[41] Dommaschk, M.; Echavarren, J.; Leigh, D. A.; Marcos, V.; Singleton, T. A. Angew. Chem., Int. Ed. 2019, 58, 14955.

[42] De Bo, G.; Leigh, D. A.; McTernan, C. T.; Wang, S. Chem. Sci. 2017, 8, 7077.

[43] (a) Chaur, M. N.; Collado, D.; Lehn, J.-M. Chem.-Eur. J. 2011, 17, 248.

(b) Su, X.; Aprahamian, I. Chem. Soc. Rev. 2014, 43, 1963.

[44] (a) Allgeier, A. M.; Mirkin, C. A. Angew. Chem., Int. Ed. 1998, 37, 894.

(b) Praneeth, V. K. K.; Ringenberg, M. R.; Ward, T. R. Angew. Chem., Int. Ed. 2012, 51, 10228.

[45] Zahn, S.; Canary, J. W. Science 2000, 288, 1404.

[46] Mortezaei, S.; Catarineu, N. R.; Canary, J. W. J. Am. Chem. Soc. 2012, 134, 8054

[47] Mortezaei, S.; Catarineu, N. R.; Duan, X.; Hu, C.; Canary, J. W. Chem. Sci. 2015, 6, 5904.

[48] Zhang, Q.; Cui, X.; Zhang, L.; Luo, S.; Wang, H.; Wu, Y. Angew. Chem., Int. Ed. 2015, 54, 5210.

[49] (a) Kassem, S.; Lee, A. T. L.; Leigh, D. A.; Marcos, V.; Palmer, L. I.; Pisano, S. Nature 2017, 549, 374.

(b) Kelly, T. R.; Snapper, M. L. Nature 2017, 549, 336.

[50] (a) Neel, A. J.; Hilton, M. J.; Sigman, M. S.; Toste, F. D. Nature 2017, 543, 637.

(b) Huang, G.; Chen, Z.; Wei, X.; Chen, Y.; Li, X.; Zhong, H.; Tan, M. Chin. J. Org. Chem. 2020, 40, 614 (in Chinese). (黄国保, 陈志林, 韦贤生, 陈钰, 李秀英, 仲辉, 谭明雄, 有机 化学, 2020, 40, 614.)

(c) Wang, Y.; Liu, H.; Zhu, X. Acta Chim. Sinica 2020, 78, 746 (in Chinese).

(王友付, 刘航海, 朱新远, 化学学报, 2020, 78, 746.) 